\title{
Nails Involvement in Winiwarter-Buerger Disease
}

\author{
Michela V.R. Starace ${ }^{a}$ Aurora Alessandrini ${ }^{a}$ Antonella Tosti ${ }^{b}$ \\ Bianca Maria Piraccini ${ }^{\mathrm{a}}$ \\ aDepartment of Specialized, Experimental and Diagnostic Medicine, Dermatology-IRCCS, Policlinico Sant'Orsola, \\ Alma Mater Studiorum, University of Bologna, Bologna, Italy; ${ }^{\text {DD }}$. Phillip Frost Department of Dermatology and \\ Cutaneous Surgery, Fredric Brandt Endowed Professor of Dermatology, University of Miami, Miller School of \\ Medicine, Miami, FL, USA
}

\section{Established Facts}

- Buerger disease, also called thromboangiitis obliterans, is an inflammatory and occlusive process involving small and medium size arteries and veins.

- The process can result in digital amputation.

- Involvement of nails is not common.

\section{Novel Insights}

- The first signs of thromboangiitis obliterans can appear at fingernails, showing chronic paronychia, onycholysis, and nail bed erosion.

- Dermatologist can make an early diagnosis, directing the patient to a pharmacological or an endovascular treatment.

\section{Keywords}

Winiwarter-Buerger disease · Nail · Paronychia .

Leukonychia - Erosion nail bed · Vascular damage · Smoke

\section{Abstract}

Introduction: Buerger disease, or thromboangiitis obliterans, is an inflammatory and occlusive process involving small and medium size arteries and veins, which generally affects the lower limbs of young adult male with the habit of smoking. Case Presentation: This paper reports 2 patients who developed nail lesions as the first sign of Buerger disease. Conclusion: Signs and symptoms of Buerger's disease are secondary to the inflammatory process and arterial occlusion which results in severe ischemia. Involvement of nails is not common, but we found 2 different clinical features which have not been previously reported in the literature: chronic paronychia, and proximal leukonychia or onycholysis and nail bed erosion.

(c) 2021 The Author(s)

Published by S. Karger AG, Basel
C 2021 The Author(s).

Published by S. Karger AG, Basel

This article is licensed under the Creative Commons Attribution 4.0 International License (CC BY) (http://www.karger.com/Services/ OpenAccessLicense). Usage, derivative works and distribution are permitted provided that proper credit is given to the author and the original publisher.
Correspondence to:

Aurora Alessandrini, aurora.alessandrini3@unibo.it 


\section{Introduction/Literature Review}

Buerger disease is an inflammatory and occlusive process involving small and medium size arteries and veins, which generally affects the lower limbs of young adult male with the habit of smoking. Also called thromboangiitis obliterans, this nonatherosclerotic, segmental, inflammatory, and occlusive process often results in severe disability and digital amputation [1]. Involvement of nails is not common as the first clinical sign, and its differentiation from other causes of nail lesions is difficult. This paper reports 2 patients who developed nail lesions as the first sign of Bueger disease. Patients have given their written informed consent to publish their case (including publication of images).

\section{Case Report 1}

A 46-year-old Caucasian man was referred to our Department with a suspicion of onychomycosis involving the second and third fingernail. Clinical examination (Fig. 1a) revealed a paronychia in both fingers and a proximal leukonychia and hemorrhagic lesion at the fingertip of the third finger. The affected fingers were cold to the touch. The history revealed that the nail lesions had been present for 6 months and were associated with paresthesia and pain and the touch are cold. The patient also developed vice to smoke 20 cigarettes a day for many years and suffered from several episodes of left ilium pain while walking. Radiological examination of his right hand was normal. Vascular examination showed bilateral anterior and posterior tibial pulses, and the left popliteal pulse was not palpable. A vascular murmur was present in superficial and common femoral arteries. Laboratory analysis demonstrated an increased VES and fibrinogen. Arterial Dopplers of his right upper limb showed a decreased flow in the arterial palmar arch, and a very decreased flow in the first, third, and fourth interdigital arteries, as well as an absent flow in the second interdigital artery. All digital arteries showed absent flow. The patient was finally transferred to the Angiology Department with the diagnosis of Buerger disease in accord to Shionoya's criteria [2]. Treatment with Prostavasin (60 mcg a day) for 11 days and Endoprost ( $0.05 \mathrm{mg}$ a day) for 4 days did not produce significant improvement of pain and symptoms. Examination after 2 months (Fig. 1b) revealed onychomadesis and nail bed ulceration. The patient reduced but did not stop smoking.

\section{Case Report 2}

A 65-year-old Caucasian man was referred to our Department with a suspicion of malignant nail tumor involving the third fingernail. Clinical examination (Fig. 2) revealed onycholysis with
Fig. 1. a The affected nails at the first clinical examination, showing chronic paronychia in both fingers, proximal leukonychia and a hemorrhagic lesion at the fingertip of the third finger (the hole in the third nail finger is artificially made). $\mathbf{b}$ The affected nails at the second clinical examination 2 months later, showing onychomadesis and nail bed ulceration.

Fig. 2. A 65-year-old Caucasian man with onycholysis, erosion of the nail bed, presence of purulent exudate and marked chronic paronychia of the third fingernail.
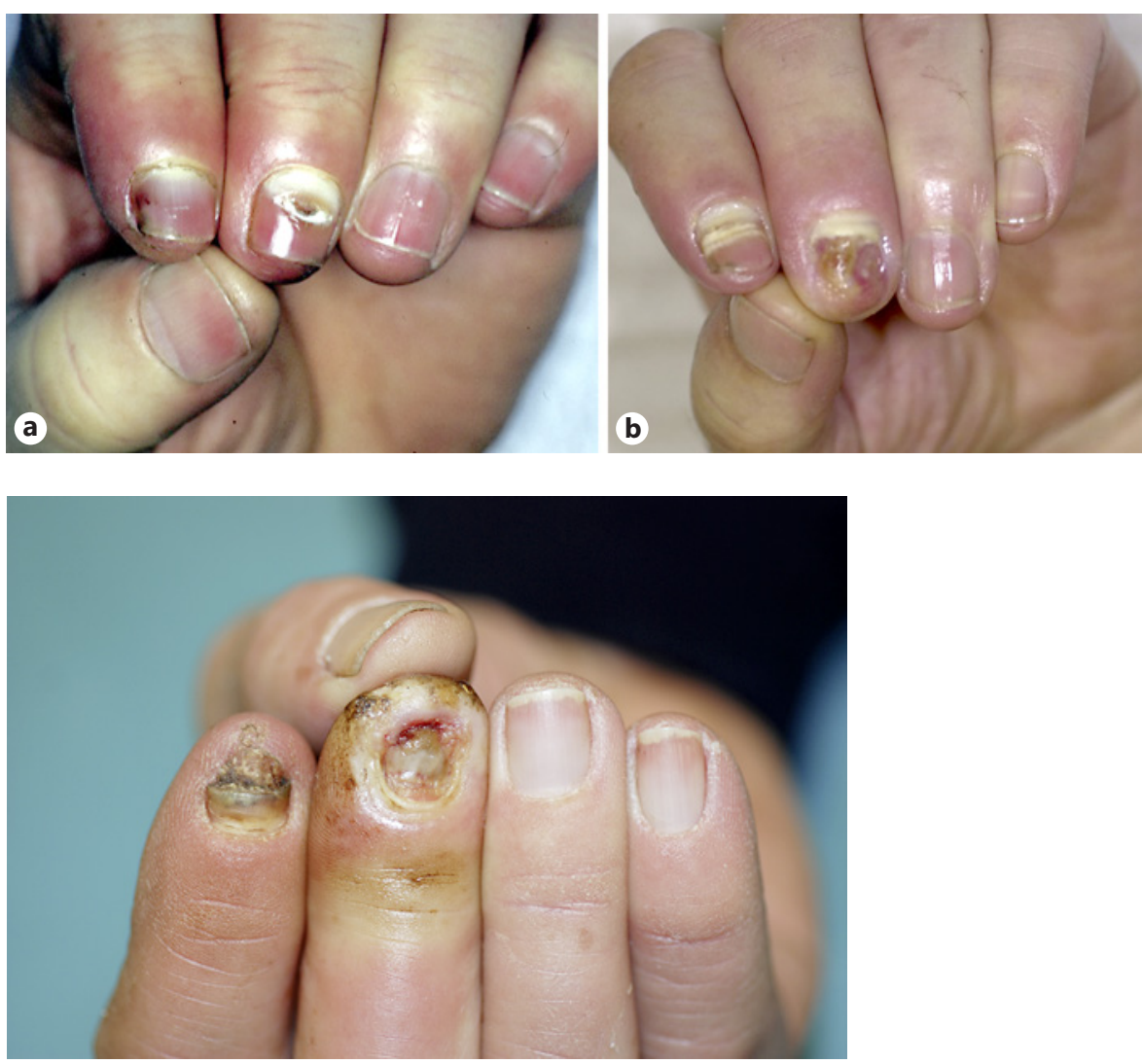

Nails as an Indicator of a Vascular Disorder
Skin Appendage Disord 2022;8:142-145 DOI: $10.1159 / 000518982$ 
erosion of the nail bed, presence of purulent exudate, with marked chronic paronychia in this fingernail, with a similar process also in the second fingernail, which presented onycholysis and exudation. Touching them, the fingers were cold. He declared that the lesion was present in the last 10 months. Medical history also confirms that the patient had been a smoker for many years. Radiological examination was normal. Vascular examination with Dopplers and angiography confirmed our suspicion of Buerger disease. $\mathrm{He}$ moved to another city, so unfortunately, we do not have data about the follow-up.

\section{Discussion}

Thromboangiitis obliterans is an uncommon, but not extremely rare vasculitis, which occurs more often in Asian countries and in young adult men smokers [3]. Its specific etiology remains obscure, but there are secondary etiologic factors which predispose to the disease, 1 such as young age, male sex, oriental race, hereditary factors [4], autoimmune process [5], muscular occupation, changes in blood, drugs and poisons [6-8], and especially smoking. Burger's disease has rarely been reported in nonsmokers and frequently improve associated factor. Perhaps a genetically controlled hypersensitivity to tobacco, due to a particular HLA phenotype, along with an impairment of sympathoadrenal function and an altered peripheral adrenergic response to cigarette smoking [9] are the trigger elements of this disease. This is then accelerated by various internal and external factors.

Signs and symptoms are secondary to the inflammatory process and arterial occlusion which results in severe ischemia. While the most frequent [10-12] are present in the lower extremely and visceral, our report underscores the importance of clinical manifestations in the nail [1315]. We found 2 clinical signs, chronic paronychia and proximal leukonychia, which have not been previously reported in Burger's disease literature. Commonly, in the first stage of the disease severe ischemia causes delay in nail growth and a distortion of the nail plate, which becomes thick, rough, and transversally or longitudinally ridged. The nail bed characteristically presents as splinter hemorrhages hidden by the thick nail. This abnormal nail growth may lead to claw nails (onychogryphosis), but an improvement of blood supply would allow the nail to return to normal. As the process advances, nail plates may show Beau's lines, shedding, loosening, and permanent distortion [8]. When healing takes place, the nail usually remains distorted or may be completely lost and replaced by scar tissue [16].
In our cases, the first signs of thromboangiitis obliterans appeared at fingernails, with chronic paronychia and leukonychia in the first case, onycholysis and nail bed erosion in the second 1 . In our opinion, chronic paronychia and erosion could be a consequence of digital ischemia and inflammation producing trophic lesion in periungual tissues and nail bed and leukonychia could be consequence of abnormalities in keratinization due to nail matrix damage. In our patients, the fingers involved are the first and second fingers, and we postulate that the explanation is due because these fingers are the ones used to hold the cigarette when 1 smokes. We would like to stress the nails should be examined in patients affected by vascular disease because their changes may represent the first sign of blood supply impairment.

Dermatologist can make an early diagnosis, increasing the success of pharmacologic treatment and endovascular revascularization, reducing the risk of minor and major amputation $[17,18]$. In particular, the association between chronic paronychia, leukonychia, nail bed erosion, and cold fingers should aware the physician to the possibility of a peripheral vascular disease $[19,20]$.

\section{Statement of Ethics}

Subjects have given their written informed consent to publish their case (including publication of images). Ethical approval was not required for this study in accordance with national guidelines.

\section{Conflict of Interest Statement}

The authors have no conflicts of interest to declare.

\section{Funding Sources}

No funding sources to declare.

\section{Author Contributions}

Every author contributed equally in the manuscript, for writing and editing the paper.

\section{Data Availability Statement}

All data generated during this study are included in this article. Further enquiries can be directed to the corresponding author. 


\section{References}

1 Mishima Y. Thromboangioiitis obliterans (Buerger's disease). Int J Cardiol. 1996; 54(Suppl 1):S185-7.

2 Shionoya S. Patholology. In: Shionoya S, editor. Buerger's disease. Nagoya, Japan: University of Nagoya Press; 1990. p. 57-79.

3 Tanaka K. Pathology and pathogenesis of Buerger's disease. Int J Cardiol. 1998;66(Suppl 1):S237-42.

4 Numano F. Takayasu arteritis, Buerger disease and inflammatory abdominal aortic aneurysm: is there a common pathway in their pathogenesis? Int J Cardiol. 1998;66(Suppl 1): 5-10.

5 Kurata A, Franke FE, Machinami R, Schulz A. Tromboangoiitis obliterans: classic and new morphological features. Virchows Arch. 2000;436:59-67.

6 Marder VJ, Mellinghoff IK. Cocaine and Buerger disease: is there a pathogenetic association? Arch Intern Med. 2000;160:2057-60.

7 Bozkurt AK. The role of cocaine in the etiology of Buerger disease is questionable. Arch Intern Med. 2001;161:486.
8 Noel B. Burger disease or arsenic intoxication? Arch Intern Med. 2001;161:1016.

9 RonconAlbuquerque R, Serrao P, ValePereira $\mathrm{R}$, CostaLima J, RonconAlbuquerque R Jr. Plasma catecholamines in Burger's disease: effect of sigarette smoking and surgical sympathectomy. Eur J Vasc Endovasc Surg. 2000; 24:338-43.

10 Kobayashi M, Kurose K, Kobata T, Hida K, Sakamoto S, Matsubara J. Ischemic intestinal involvement in a patient with Burger disease: case report and literature review. J Vasc Surg. 2003;38:170-4.

11 Gaudin JL, Vallas M, Genin G, Souquet JC, Bel A. False aneurysm of the hepatic artery revelaled by hemobilia in Burger's disease: an incidental association? Gastroentrol Clin boil. 1993; 17:6089.

12 Filis KA, Bastounis EA. Regarding "ischemic intestinal involvement in a patient with Buerger disease: case report and literature review". J Vasc Surg. 2004;39:486.

13 Drake ME Jr. WiniwarterBurger disease ("thromboangioiitis obliterans") with cerebral involvement. JAMA. 1982;248:1870-2.
14 Giblin WJ, James WD, Benson PM. Buerge's disease. Int J Dermatol. 1989;28:672-3.

15 Edwards EA. Nail changes in functional and organic arterial disease. N Engl J Med. 1948; 239:362-5.

16 Samman PD, Strickland B. Abnormalities of the finger nails associated with impaired peripheral blood supply. Br J Dermatol. 1962;74: 165-73.

17 Cacione DG, Macedo CR, do Carmo Novaes F, Baptista-Silva JC. Pharmacological treatment for Buerger's disease. Cochrane Database Syst Rev. 2020 May 4;5(5):CD011033.

18 Del Conde I, Peña C. Buerger disease (thromboangiitis obliterans). Tech Vasc Interv Radiol. 2014 Dec;17(4):234-40.

19 Ohta T, Ishioashi H, Hosaka M, Sugimoto I. Clinical and social consequences of Buerger disease. J Vasc Surg. 2004;39:176-80.

20 Nakajima N. The change in concept and surgical treatment on Buerger's disease-personal experience and review. Int J Cardiol. 1998; 66(Suppl 1):S273-80. 